\title{
Practical Review of Biologics in Chronic Rhinosinusitis With Nasal Polyps
}

\author{
Ki-II Lee, MD, PhD', Gwanghui Ryu, MD², Shin Hyuk Yoo, MD $^{3}$, \\ Yong Min Kim, MD, $\mathrm{PhD}^{4}$, Ji-Hun Mo, MD, $\mathrm{PhD}^{3}$, and Seung-Heon Shin, MD, $\mathrm{PhD}^{5}$ \\ ${ }^{1}$ Department of Otorhinolaryngology-Head and Neck Surgery, Konyang University College of Medicine, Daejeon, Republic of Korea \\ ${ }^{2}$ Department of Otorhinolaryngology-Head and Neck Surgery, Soonchunhyang University College of Medicine, Cheonan, Republic of Korea \\ ${ }^{3}$ Department of Otorhinolaryngology-Head and Neck Surgery, Dankook University College of Medicine, Cheonan, Republic of Korea \\ ${ }^{4}$ Department of Otorhinolaryngology-Head and Neck Surgery, Chungnam National University School of Medicine, Daejeon, Republic of Korea \\ ${ }^{5}$ Department of Otorhinolaryngology-Head and Neck Surgery, School of Medicine, Catholic University of Daegu, Daegu, Republic of Korea
}

Well-characterized in chronic rhinosinusitis, type 2 inflammation is frequently associated with nasal polyps, comorbid asthma, and nonsteroidal anti-inflammatory drug hypersensitivity. Despite medical and surgical treatment, it recurs in a significant proportion of patients. Thus, severe uncontrolled type 2 chronic rhinosinusitis with nasal polyps is the most difficult-to-treat phenotype of chronic rhinosinusitis. Recently, dupilumab, a monoclonal antibody against IL-4 receptor $\alpha$, and omalizumab, a monoclonal antibody against immunoglobulin E, were approved for patients with chronic rhinosinusitis with nasal polyps in the United States, Europe, and Korea. Therefore, rhinologists should understand novel biologics and their use. Here, we provide a literature review of several biologics with their indications, effectiveness, and safety.

Keywords: Biologics; Sinusitis; Immunology; Nasal polyps; Interleukin-4 receptors.

\section{INTRODUCTION}

Chronic rhinosinusitis (CRS) is an inflammatory disease of the upper respiratory tract accounting for most outpatient otolaryngology visits. ${ }^{1)}$ The prevalence of CRS is $14 \%$ in the United States, $11 \%$ in Europe, $7 \%$ in Korea, and 5\% in Cana$\mathrm{da}$, and that of CRS with nasal polyps (CRSwNP) is $4 \%$ in the United States, $2 \%-4 \%$ in Europe, 3\% in Korea, and $0.5 \%$ in China. ${ }^{2-5)}$ However, the actual prevalence of CRS is estimated to be higher because of the lack of systematic epidemiological studies on CRS and regional differences in the diagnostic criteria. ${ }^{3)}$ CRSwNP leads to rhinologic symptoms, such as na-

Received: July 29, 2021 Revised: September 29, 2021

Accepted: October 12, 2021

Address for correspondence: Ji-Hun Mo, MD, PhD, Department of Otorhinolaryngology-Head and Neck Surgery, Dankook University College of Medicine, 119 Dandae-ro, Dongnam-gu, Cheonan 31116, Republic of Korea

Tel: +82-41-550-6480, Fax: +82-41-556-1090, E-mail: jihunmo@gmail.com Address for correspondence: Seung-Heon Shin, MD, PhD, Department of Otorhinolaryngology-Head and Neck Surgery, School of Medicine, Catholic University of Daegu, 33 Duryugongwon-ro 17-gil, Nam-gu, Daegu 42472, Republic of Korea

Tel: +82-53-650-4530, Fax: +82-53-650-4533, E-mail: hsseung@cu.ac.kr This is an Open Access article distributed under the terms of the Creative Commons Attribution Non-Commercial License (https://creativecommons.org/licenses/by$\mathrm{nc} / 4.0$ ) which permits unrestricted non-commercial use, distribution, and reproduction in any medium, provided the original work is properly cited. sal congestion, purulent discharge, and post-nasal drip; and other symptoms that reduce the quality of life, such as chronic fatigue, reduced sense of taste and attention, and sleep disorders. ${ }^{6}$ In particular, reduced sense of smell, which is one of the rhinologic symptoms of CRS, is reported to be highly associated with neurological disorders, such as depression and anxiety. ${ }^{7)}$ Additionally, type 2 inflammation is highly associated with CRSwNP and is often accompanied by asthma, allergic rhinitis, atopic dermatitis, and hypersensitivity to non-corticosteroidal anti-inflammatory drugs (NSAIDs) clinically. ${ }^{8) 9}$ The incidence of type 2 inflammation in CRSwNP is reported to be $80 \%$ in the Western countries and has been increasing in Asian countries. ${ }^{10) 11)}$ However, common treatments of CRSwNP_such as saline irrigation, topical corticosteroids, and antibiotics-lack effectiveness and recurrence is common after endoscopic sinus surgery, requiring a reoperation. ${ }^{12) 13 \text { ) }}$ While CRSwNP responds well to systemic corticosteroids, long-term use of systemic corticosteroids is impractical because of their side effects. ${ }^{14)}$ Although CRSwNP often does not respond to common treatments, there are no practical alternatives to these treatments.

Recently, the use of novel biologics targeting type 2 inflammation via immunological mechanisms have been consid- 
ered as a viable treatment option for severe uncontrolled type 2 CRSwNP. Here, we present a literature review on the immunological and clinical characteristics of biologics targeting severe uncontrolled type $2 \mathrm{CRSwNP}$ and discuss the rhinologic considerations for physicians.

\section{CRSwNP AND TYPE 2 INFLAMMATION}

\section{Pathophysiology of type 2 inflammation}

Nasal polyps commonly result from type 2 inflammation mediated by type 2 cytokines in the upper respiratory tract. Type 2 inflammation was previously referred to as "T helper cell 2 (Th2)-mediated inflammation," since it involves an acquired immune response mediated by Th2 cells. However, following the discovery that cells involved in innate immunity play an important role in Th2 cell-mediated inflammation, it was later renamed as "type 2 inflammation." ")15)

The most important inflammatory cytokines in type 2 inflammation are interleukin (IL)-4, IL-13, and IL-5. ${ }^{8) 16}$ The respiratory mucosa releases epithelial-derived cytokines, such as IL-25, IL-33, and thymic stromal lymphopoietin (TSLP), in response to the presence of foreign bacteria, viruses, or irritants, which then stimulate type 2 innate lymphoid cells (ILC2) to secrete major cytokines, such as IL-5 and IL-13. Researchers have recently discovered that ILC2 produces IL-4, which induces the differentiation of Th0 (naïve T cells) to Th2 cells. IL-4 and IL-13, which are released later, induce class switching of B cell isotypes to produce immunoglobulin E (IgE). Furthermore, they inhibit tissue plasminogen activator, thus interfering with fibrinolysis and inducing fibrin cross-linking via M2 macrophage polarization, which subsequently leads to tissue remodeling and polypogenesis. IL- 4 induces Th2 cell differentiation and IL-13 acts on epithelial cells to induce goblet cell hyperplasia and increase mucous secretions. IL-5 is involved in the activation, recruitment, and survival of eosinophils, which plays an essential role in type 2 inflammation (Fig. 1).

\section{Severe uncontrolled type 2 CRSwNP}

The European Forum for Research and Education in Allergy and Airway Diseases (EUFOREA), which is an international nonprofit organization, published an expert board meeting

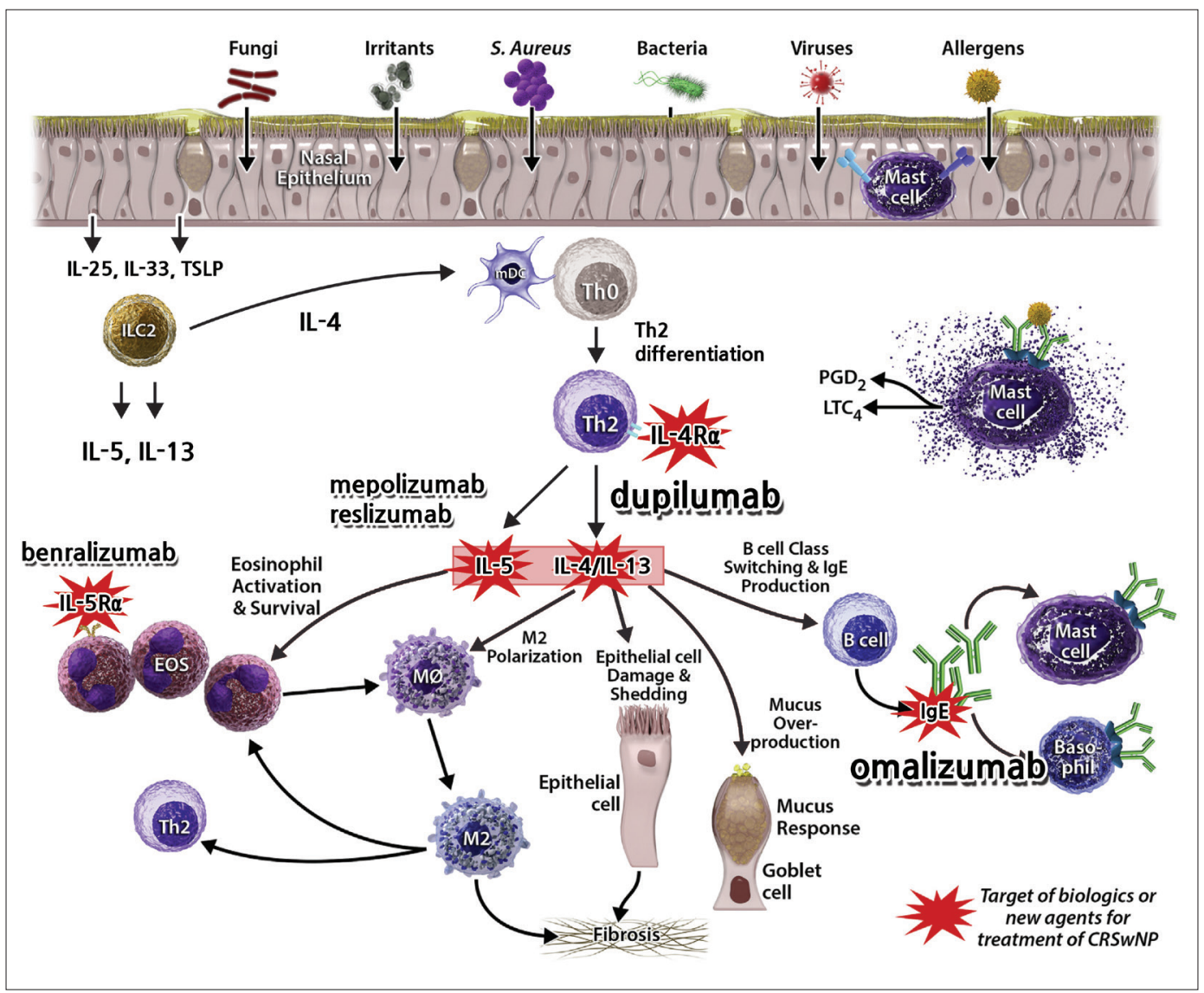

Fig. 1. Immunological pathomechanism and biologics according to their targets in type 2 inflammation. Red starbursts focus the targets of novel biologics for chronic rhinosinusitis with nasal polyps (CRSwNP). Modified from Laidlaw et al. Ann Allergy Asthma Immunol 2020; 124(4): 326-32, with permission of Elsevier. ${ }^{55)}$ 
on severe uncontrolled type 2 CRSwNP. ${ }^{17)}$ They defined "uncontrolled" CRSwNP as the condition when symptoms persist or recur within 2 years after long-term use of topical steroids or after at least one course of systemic corticosteroid treatment or surgery. They also defined "severe" disease as CRSwNP with nasal polyp scores of at least 4 out of 8 , requiring additional treatments apart from topical steroids because of main symptoms, such as reduction of smell/taste, nasal congestion, and nasal discharge, and accessory symptoms, such as post-nasal drip and facial pain/pressure, persisting after the initial treatment.

By definition, severe uncontrolled CRSwNP is associated with type 2 inflammation and refers to cases of CRS in which the treatment response to pharmacotherapy or surgery is poor. Hence, a new treatment strategy using an immunological approach must be developed.

\section{Type 2 inflammatory shift}

The association between type 2 inflammation and CRSwNP is reported to be $15 \%-85 \%$, varying between regions and ethnicities. ${ }^{10) 18)}$ Wang et al. ${ }^{18)}$ reported high levels of type 2 inflammatory cytokines in patients from Benelux, Germany, Australia, and Japan; a mixed profile of type 1, type 2, and type 3 cytokines in patients from Beijing, China; and low levels of type 2 cytokines in those from Cheongdu, China.

Zhang et al. ${ }^{11)}$ reported regional and racial differences in nasal polyps and an eosinophilic shift, indicating a high relative prevalence of type 2 inflammation in Asian countries. These immunological changes have also been observed in Korea. $^{19)}$

Karin et al. ${ }^{20)}$ analyzed the levels of IgE, eosinophil cationic protein (ECP), and IL-5 in Belgian patients at two different time points and reported an increased incidence of type 2 inflammation in the latter group compared to that in the earlier group. These immunological changes in CRSwNP have also been confirmed in Western countries and show a global trend. Based on the immunological mechanism of CRS, the eosinophilic shift was renamed as "type 2 inflammatory shift." ${ }^{20)}$ This shift mean that there will be more severe cases where CRS is difficult to treat in the future.

\section{GENERAL TREATMENTS FOR CRSwNP}

\section{Treatment guideline for CRSwNP}

The International Consensus Statement on Allergy and Rhinology: Rhinosinusitis 2021 (ICAR statement) by the American Rhinologic Society recommends the use of a topical corticosteroid spray or corticosteroid nebulizer, corticosteroid irrigation, and short-term administration of systemic corticosteroids as the primary care for CRSwNP. ${ }^{21)}$ Corticosteroideluting implants, macrolide antibiotics, and leukotriene inhibitors may also be considered for the treatment of CRSwNP (Table 1). Surgery could be considered if a patient does not respond to pharmacotherapy within 3-4 weeks, depending on the Sino-Nasal Outcome Test-22 (SNOT-22) scores.

\section{Limitations of general treatments}

Common medications or surgical procedures for CRS have limitations in clinical settings. Nasal irrigation with saline or short-term use of topical steroids, leukotriene inhibitors, or antibiotics, which are commonly used to treat CRSwNP, lack clinical effectiveness and only play a role in maintenance at a time before the formation of nasal polyps. The effects of systemic corticosteroids used to treat loss of smell are not sustained and are reverted once the patient stops using them. ${ }^{22)}$ Furthermore, systemic corticosteroids cannot be prescribed for long-term use as they can lead to side effects, such as hyperglycemia, hypertension, mood swings, and sleep disorders,

Table 1. General treatment recommendation on CRSwNP according to ICAR 2021 statement $^{21)}$

\begin{tabular}{|c|c|c|c|}
\hline Treatment & Grade* & Policy & Clinical limitation on CRSwNP \\
\hline Intranasal corticosteroid spray & A & Strong recommendation & Minimal clinically important difference on the effect \\
\hline Oral corticosteroids & A & Strong recommendation & $\begin{array}{l}\text { Short-term use } \\
\text { Significant side effects such as weight gain, anxiety, } \\
\text { depression, adrenal suppression, hypertension, } \\
\text { immunosupression, diabetes, osteoporosis, kidney stones }\end{array}$ \\
\hline Nasal saline irrigation & No study & Option & No RCTs on general treatment of CRSwNP \\
\hline Corticosteroid-eluting implants & A & Option & Not available in Korea \\
\hline Macrolide antibiotics & B & Option & Potential cardiotoxicity \\
\hline Non-macrolide antibiotics & $\mathrm{B}(-)$ & Recommendation against & Preponderance of harm over benefits \\
\hline Anti-leukotrienes & A & Option & Limited benefit with intranasal corticosteroid spray \\
\hline Topical antibiotics & $\mathrm{A}(-)$ & Recommendation against & Relative harm over benefit \\
\hline
\end{tabular}

*Grade: A, well-designed RCTs; B, RCTs with minor limitation; C, observational studies; and D, expert opinion or case reports. ICAR, International Consensus Statement on Allergy and Rhinology; CRSwNP, chronic rhinosinusitis with nasal polyps; RCTs, randomized controlled trials 
as well as increased risk of fracture due to osteoporosis, infection due to immunosuppression, ophthalmological problems (e.g., cataract and glaucoma), obesity due to hormonal changes, and neuropsychological problems (e.g., depression and anxiety). ${ }^{14)}$ Therefore, the European Position Paper on Rhinosinusitis and Nasal Polyps 2020 (EPOS 2020), ${ }^{23)}$ ICAR 2021, ${ }^{21)}$ and Global Initiative for Asthma (GINA) 2019 guideline ${ }^{24)}$ mutually recommend against the continuous use of systemic corticosteroids considering their side effects.

Although surgical intervention is more effective than pharmacotherapy, the postoperative recurrence and reoperation rates of CRS are high at $40 \%-79 \%$ and $36 \%$, respectively. ${ }^{922) 25 / 26)}$ These high rates suggest that although the surgical removal of polyps may manage to ventilate the paranasal sinuses and restore mucociliary movements, it cannot completely suppress inflammation because severe uncontrolled CRSwNP is the result of an immunological response associated with type 2 inflammation.

\section{NOVEL BIOLOGICS FOR SEVERE UNCONTROLLED TYPE 2 CRSWNP}

\section{Mechanism of action and approval status}

Biologics used for CRSwNP target cytokines and cells that mediate type 2 inflammation (Fig. 1). ${ }^{27)}$ Monoclonal antibodies targeting IL-5, which regulates eosinophils involved in type 2 inflammation, include mepolizumab and reslizumab, and the biologics inhibiting IL- 5 receptor $(R) \alpha$, which is an eosinophil receptor, include benralizumab. ${ }^{17)}$ Omalizumab inhibits free IgE, which regulates cell differentiation into baso- phils and mast cells by binding to the FceRI. ${ }^{23228)}$ In signaling pathways, type I receptor consists of IL- $4 \mathrm{R} \alpha$ and common gamma chain, binding to IL-4 only while type II receptor consists of IL- 4 R $\alpha$ and IL-13R $\alpha 1$, binding to both IL- 4 and IL-13. The IL-4R $\alpha$ subunit exists in type I and type II receptors commonly. Dupilumab is a monoclonal antibody that targets IL$4 \mathrm{R} \alpha$ to inhibit both IL-4 and IL-13 simultaneously. By inhibiting IL-4 and IL-13, which are the major upstream cytokines in the type 2 inflammatory pathway, dupilumab can also downregulate the chemotaxis of eosinophils in the periphery. ${ }^{27)}$

The use of dupilumab for CRSwNP has been approved by the US Food and Drug Administration and the European Medicines Agency. In Korea, it was approved by the Ministry of Food and Drug Safety in the first half of 2021. Omalizumab was approved in the United States and Europe in 2020, and Korea in the first half of 2021. Reslizumab, mepolizumab, and benralizumab have been approved for severe asthma in and outside Korea, but not for CRSwNP (Table 2).

\section{Indications and treatment response of biologics}

Up to date, there is no clinical consensus on indication of biologics for CRSwNP. Fujieda et al. ${ }^{29)}$ reported that dupilumab produce consistent improvement in symptoms irrespective of eosinophilic status of CRSwNP through the posthoc analysis of dupilumab phase 3 study. Therefore, they concluded that blood eosinophil level may not be an appropriate biomarker for biologics efficacy in CRSwNP.

Meanwhile, according to the EPOS $2020{ }^{23)}$ biologics can be indicated for CRSwNP in patients with bilateral nasal polyps with or without a previous history of surgery satisfying at least

Table 2. Current approval status for novel biologics on type 2 inflammation (Jun 30, 2021)

\begin{tabular}{|c|c|c|c|c|}
\hline Biologics & Target action & Korea (MFDS) & United States (FDA) & Europe (EMA) \\
\hline $\begin{array}{l}\text { Dupilumab } \\
\text { (Dupixent }^{\mathbb{R}} \text { ) }\end{array}$ & Block IL-4R $\alpha$ & $\begin{array}{l}\text { CRSwNP (Mar, 2021) } \\
\text { Atopic dermatitis (Mar, 2018) } \\
\text { Asthma (Apr, 2020) }\end{array}$ & $\begin{array}{l}\text { CRSwNP (Jun, 2019) } \\
\text { Atopic dermatitis (Mar, 2017) } \\
\text { Asthma (Oct, 2018) }\end{array}$ & $\begin{array}{l}\text { CRSwNP (Oct, 2019) } \\
\text { Atopic dermatitis (Jul, 2017) } \\
\text { Asthma (May, 2019) }\end{array}$ \\
\hline $\begin{array}{l}\text { Omalizumab } \\
\left.\text { (Xolair }^{\circledR}\right)\end{array}$ & Bind free $\operatorname{IgE}$ & $\begin{array}{l}\text { CRSwNP (Apr, 2021) } \\
\text { Asthma (May, 2007) } \\
\text { Chronic idiopathic urticaria } \\
\text { (Sep, 2017) }\end{array}$ & $\begin{array}{l}\text { CRSwNP (Dec, 2020) } \\
\text { Asthma (Jun, 2003) } \\
\text { Chronic idiopathic urticaria } \\
\text { (May, 2014) }\end{array}$ & $\begin{array}{l}\text { CRSwNP (Aug, 2020) } \\
\text { Asthma (Oct, 2005) } \\
\text { Chronic idiopathic urticaria } \\
\text { (Mar, 2014) }\end{array}$ \\
\hline $\begin{array}{l}\text { Mepolizumab } \\
\left(\text { Nucala }^{\circledR}\right)\end{array}$ & Inhibits IL-5 & Asthma (Apr, 2016) & $\begin{array}{l}\text { Asthma (Nov, 2015) } \\
\text { Churg-Strauss syndrome } \\
\text { (Dec, 2017) } \\
\text { Hypereosinophilic syndrome } \\
\text { (Sep, 2020) }\end{array}$ & Asthma (Sep, 2015) \\
\hline $\begin{array}{r}\text { Reslizumab } \\
\left(\text { Cinqair }^{\circledR}\right)\end{array}$ & Inhibits IL-5 & Asthma (Sep, 2017) & Asthma (Mar, 2016) & Asthma (Aug, 2016) \\
\hline $\begin{array}{l}\text { Benralizumab } \\
\left(\text { Farsenra }^{\circledR}\right)\end{array}$ & Block IL-5R $\alpha$ & Asthma (Jun, 2019) & Asthma (Nov, 2017) & Asthma (Jan, 2018) \\
\hline
\end{tabular}

MFDS, Ministry of Food and Drug Safety; FDA, U.S. Food and Drug Administration; EMA, European Medicines Agency; CRSwNP, chronic rhinosinusitis with nasal polyps; IL, interleukin; Churg-Strauss syndrome, eosinophilic granulomatosis with polyangiiti 
three of the following five conditions: 1) type 2 inflammation ( $\geq 10$ tissue eosinophils/high-power field, $\geq 250$ blood eosinophils/ $\mu \mathrm{L}$, or total $\operatorname{IgE} \geq 100 \mathrm{kU} / \mathrm{L}$ ); 2) need for at least two courses of systemic corticosteroids or $\geq 3$ months of systemic corticosteroid use; 3 ) reduced quality of life (SNOT-22 scores $\geq 40$ ); 4) reduced sense of smell in the smell test; and 5) asthma requiring inhaled corticosteroids. ${ }^{23)}$

Additionally, the EPOS 2020 presented five evaluation criteria for assessing the treatment response of biologics: 1) reduced nasal polyp size; 2 ) reduced need for systemic corticosteroids; 3) improved quality of life; 4) improved sense of smell; and 5) reduced impact of co-morbidities. ${ }^{23)}$ The treatment responses are graded as "excellent" (all five criteria satisfied), "moderate" (3-4 criteria satisfied), "poor" (1-2 criteria satisfied), or "no response" ( 0 criteria satisfied). Treatment should be discontinued if "no response" is observed in any of the criteria.

According to the EUFOREA expert board meeting, ${ }^{17)} 6$ months after initiation of therapy had been proposed as the time point to evaluate the treatment response for whether to continue biologics treatment or whether to perform surgery if it does not work.

Meanwhile, Table 3 summarizes the results of previous clinical trials on biologics for CRSwNP.

\section{Dupilumab (Dupixent ${ }^{\circledR}$ )}

Dupilumab is one of the two drugs approved for CRSwNP in Korea, the United States, and Europe. Bachert et al. ${ }^{30)}$ reported a significant reduction in nasal polyp scores in an treatment group (dupilumab+topical steroids) when compared to the control group (topical steroids) in their 16-week randomized controlled trial analyzing 60 patients with CRSwNP.

A large-scale multinational, multi-center study systematically analyzing the long-term effects and safety of dupilumab (LIBERTY SINUS-24 and -52) was later published. ${ }^{31)}$ The patients included in the study were diagnosed with severe uncontrolled type 2 CRSwNP based on their previous surgical history, nasal polyp scores, Lund-Mackay (LM) computed tomography scores, SNOT-22 scores, sense of smell, and comorbidities. The patients were divided into two groups: SINUS-24 group $(n=276)$, in which the patients were subcutaneously administered dupilumab every 2 weeks for 6 months (24 weeks), and SINUS-52 group ( $\mathrm{n}=448$ ), which received dupilumab every 2 weeks for 6 months and thereafter every 1 month (4 weeks) for an additional 6 months. The treatment groups were compared with the control group, which received topical steroids at different time points. The least-square mean differences or the mean change from baseline in the nasal polyp, nasal congestion, and LM scores after 6 months (24 weeks) of treatment (SINUS-24) were -2.06 (95\% confidence interval [CI] -2.43 to $-1.69, \mathrm{p}<0.0001),-0.89$ (95\% CI -1.07 to -0.71 ,

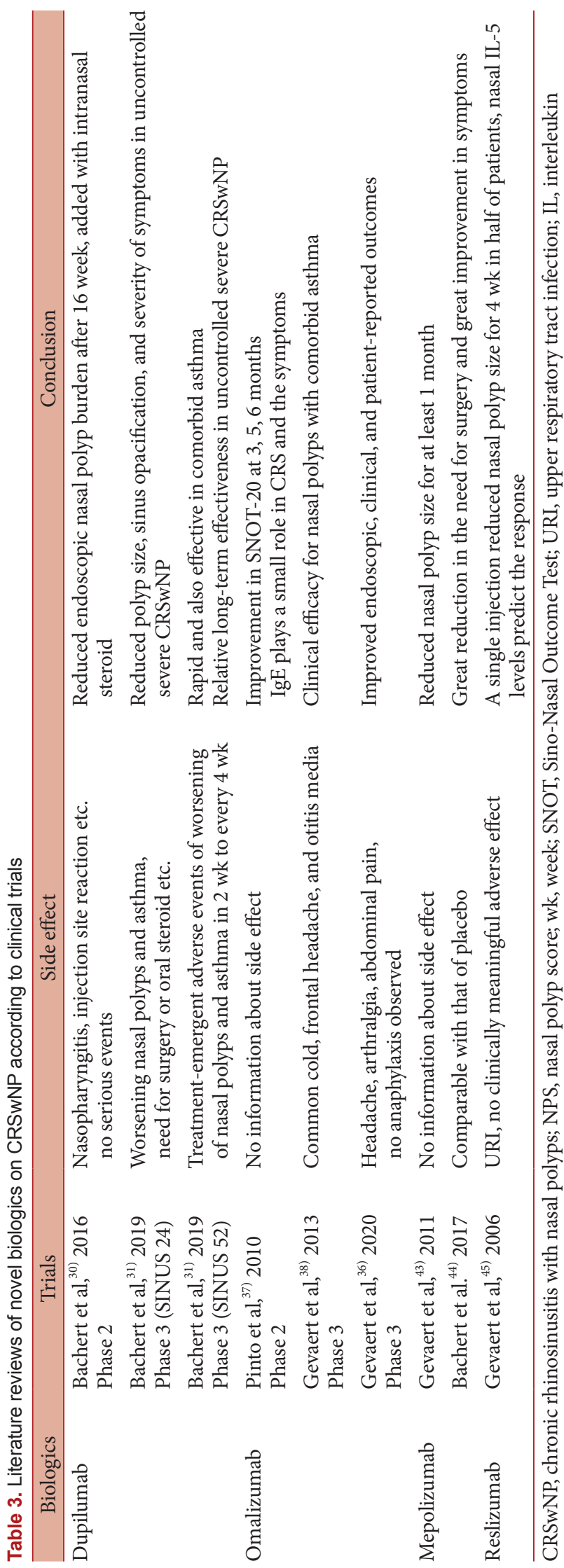


$\mathrm{p}<0.0001)$, and -7.44 (95\% CI -8.35 to $-6.53, \mathrm{p}<0.0001)$, respectively, indicating that dupilumab is significantly superior to the topical steroids. The least-square mean differences in the respective scores after 24 weeks of SINUS-52 study were -1.80 ( $95 \%$ CI -2.10 to $-1.51, \mathrm{p}<0.0001$ ), -0.87 (95\% CI -1.03 to -0.71 ), and -5.13 ( $95 \% \mathrm{CI}-5.80$ to $-4.46, \mathrm{p}<0.0001$ ), respectively, indicating that long-term administration of biologics was also effective. Interestingly, no significant difference was found according to the administration interval ( 2 weeks vs. 1 month) between 6 months and 1 year of treatment, indicating that an administration interval of 1 month may be considered during the maintenance period. On radiologic examination, the effect of dupilumab was also observed in both paranasal sinuses after 24 weeks of treatment, and a significant improvement was observed after 52 weeks. Fast improvements were also observed following dupilumab administration. The sense of smell started to improve in the early period of treatment (within 1 month), and similar improvements were observed even in patients with comorbidities, such as asthma and hypersensitivity to NSAIDs.

Numerous studies have examined subjective improvements in symptoms following dupilumab administration in patients with severe uncontrolled type 2 CRSwNP. According to a randomized controlled study that evaluated the SNOT-22 test, a 36-Item Short-Form Health Survey, and a five-dimension EuroQoL visual analog scale questionnaire (EQ-5D VAS) among 60 patients with CRSwNP not responsive to topical steroids, the major symptoms among the patients included a reduced sense of smell, nasal congestion, purulent discharge, post-nasal drip, and reduced attention. ${ }^{32)}$ The study reported that the patients in the dupilumab group had taken fewer sick leaves than that in the control group and had shown a higher productivity in their workplaces. ${ }^{32)}$ In a study that evaluated the EQ-5D VAS among patients with asthma, significant improvements were observed in the dupilumab group compared to that in the control group. ${ }^{33)}$

Jonstam et al. ${ }^{34)}$ measured changes in biomarkers following the administration of dupilumab in patients with type 2 inflammation. They reported reductions in eotaxin-3 and total IgE levels in nasal secretions as well as reductions in ECP, eotaxin-2, eotaxin-3, IgE, and IL-13 levels in nasal polyp tissues. Takabayashi et al. ${ }^{35)}$ reported that the level of urinary leukotriene $\mathrm{E} 4$, which is a marker of leukotriene dysmetabolism, decreased while the blood eosinophil count temporarily increased in the beginning (16 weeks) after the administration of dupilumab.

Dupilumab not only improves subjective symptoms but also reduces the treatment burden. According to the aforementioned study with the SINUS-52 group, dupilumab can reduce the use of systemic corticosteroids and need for surgery by
$74.6 \%$ and $89.4 \%$, respectively. ${ }^{31)}$

Common side effects of dupilumab include nasopharyngitis, headache, nasal bleeding, and injection-site rash. Conjunctivitis, treatment-emergency eosinophilia, eosinophilic granulomatosis with polyangiitis, eosinophilia accompanied by joint pain, nasal polyps, or asthma aggravation may occur rarely. ${ }^{2131)}$

The ICAR 2021 states that dupilumab has an evidence level of A (verified by numerous randomized controlled trials) and recommends using dupilumab for severe CRSwNP that does not respond to medical or surgical treatments as its benefits outweigh its harms. ${ }^{21)}$

\section{Omalizumab (Xolair ${ }^{\circledR}$ )}

Omalizumab has been approved for use in CRSwNP in Korea. Gevaert et al. ${ }^{36)}$ conducted a phase 3 double-blind randomized controlled trial on the efficacy and safety of omalizumab. Similar to the aforementioned phase 3 trial on dupilumab (LIBERTY SINUS-24 and -52), this trial involved two groups. The study compared an experimental group, which received omalizumab and topical steroids at 2- or 4-week intervals, and a control group, which received topical steroids only. The experimental group was further divided into two groups (POLYP1 and POLYP2), following a replicate study design. A total of 138 patients in POLYP1 and 127 patients in POLYP2 showed nasal polyp scores $\geq 6$ and SNOT-22 scores of approximately 60 , indicating that severe CRSwNP significantly reduces the quality of life. The mean changes in the nasal polyp score for omalizumab vs. control from baseline at week 24 were -1.08 vs. 0.06 ( $95 \%$ CI -1.59 to $-0.69, \mathrm{p}<0.0001$ ) in POLYP1 and -0.90 vs. -0.31 ( $95 \%$ CI -1.05 to $-0.12, \mathrm{p}=0.0140$ ) in POLYP2, respectively. The mean changes for omalizumab vs. control in the nasal congestion score from baseline to week 24 were -0.89 vs. -0.35 ( $95 \%$ CI -0.84 to $-0.25, \mathrm{p}=0.0004)$ in POLYP1 and -0.70 vs. -0.20 ( $95 \%$ CI -0.80 to $-0.19, \mathrm{p}=0.0017$ ) in POLYP2, respectively. Likewise, the mean changes in SNOT-22 score for omalizumab vs. control from baseline at week 24 were -24.7 vs. -8.6 ( $95 \%$ CI -21.86 to $-10.38, \mathrm{p}<0.0001$ ) and -21.6 vs. -6.6 (95\% CI -21.26 to -8.82 , $\mathrm{p}<0.0001$ ), respectively. These results indicate that omalizumab is significantly superior to the control treatment. While other studies have also reported positive results for omalizumab in terms of clinical symptom improvements, most of them examined a small number of patients with CRSwNP and failed to systematically analyze their results. ${ }^{37-41)}$ Furthermore, there is still a lack of studies investigating the changes in biomarkers following the administration of omalizumab.

According to the abovementioned phase 3 trial, $50.4 \%$ of all patients experienced at least one side effect of omalizum$\mathrm{ab}$, but most of them were tolerable and were not specific to omalizumab as similar side effects were observed in the con- 
trol group. ${ }^{36)}$ Headache is the most common side effect of omalizumab (8\%), followed by nasopharyngitis (6\%), injection-site rash (4\%), asthma aggravation (4\%), abdominal pain (3\%), joint pain (3\%), lumbar pain (3\%), dizziness (3\%), and nasal bleeding (3\%). Anaphylaxis may occur in rare cases.

ICAR 2021 states that omalizumab has an evidence level of $B$ (verified by a small number of randomized controlled trials), which weakly recommends it as a treatment option for patients with severe CRSwNP accompanied by uncontrolled allergic asthma.

\section{Mepolizumab (Nucala $\left.{ }^{\circledR}\right)$, reslizumab (Cinqair $\left.{ }^{\circledR}\right)$, and benralizumab (Farsenra ${ }^{\circledR}$ )}

Mepolizumab and reslizumab inhibits IL-5, which is a major driver of type 2 inflammation, and benralizumab targets IL-5R $\alpha$. Therefore, mepolizumab, reslizumab, and benralizum$\mathrm{ab}$ can regulate the eosinophils activation and survival theoretically. However, few studies have systematically examined the effects of mepolizumab, reslizumab, and benralizumab on rhinosinusitis. Recently, Han et al. ${ }^{42)}$ reported that mepolizumab treatment improved nasal polyp size and nasal obstruction compared with control among 407 patients with recurrent, refractory severe CRSwNP in phase 3 study. This suggest that mepolizumab provides an effective add-on treatment option in recurred cases after the surgical treatment.

Studies have reported that mepolizumab effectively reduces the nasal polyp size ${ }^{43)}$ and risk of reoperation ${ }^{44)}$ in severe CRSwNP. ICAR 2021 states that the evidence level for mepolizumab is C and recommends it for severe CRSwNP accompanied by poorly controlled eosinophilic asthma.

Reslizumab has an evidence level of $\mathrm{C}$ based on a randomized controlled trial reporting that it reduces the nasal polyp size in patients with CRSwNP. ${ }^{45)}$ Reslizumab may be considered as a treatment option for severe CRSwNP accompanied by poorly controlled eosinophilic asthma. ${ }^{21)}$

A recent phase 3 trial (OSTRO) of benralizumab in severe CRSwNP were conducted and presented. ${ }^{46)}$ Benralizumab significantly reduced nasal polyp score and nasal blockage score compared with control group. In subgroup analysis, comorbid asthma and higher eosinophil counts were associated with greater effect and with asthma being the strongest predictor.

\section{Comparison of biologics in CRSwNP}

Hellings et al. ${ }^{47)}$ reviewed phase 2 and 3 trials on CRSwNP and compared the effects of several biologics. Although the direct comparison was not easy because of the methodological heterogeneity among the clinical trials, all biologics showed positive effects compared to the control treatment, with slight differences among themselves. Peters et al. ${ }^{48)}$ reported that dupilumab had consistently greater improvements in outcomes versus omalizumab at week 24 in indirect treatment comparison of biologics in CRSwNP. Also, greater improvements in nasal polyp scores were observed after 52 weeks of dupilumab administration compared to that with omalizumab. ${ }^{47)}$ However, even in the case of dupilumab, which achieved the greatest improvements, the nasal polyp scores were decreased from 5.64 to 3.75 in 24 weeks group and also decreased from 6.18 to 4.46 in 52 weeks group with clinically insignificant changes from baseline. In contrast, dupilumab achieved the highest improvement while mepolizumab and omalizumab achieved considerable improvements in terms of symptom improvement based on SNOT-22 scores. In detail, the SNOT-22 scores were decreased from 48.00 to 18.58 in 24 weeks group of dupilumab, from 51.01 to 23.89 in 52 weeks group of dupilumab, from 51.50 to 28.80 in mepolizumab, from 59.80 to 35.10 in POLYP1 group of omalizumab, and from 59.20 to 37.61 in POLYP2 group of omalizumab, respectively.

A previous study demonstrated that dupilumab is superior to mepolizumab and omalizumab in restoring the sense of smell. Improvements in the sense of smell were observed within 2-3 months of dupilumab administration, and the sense of smell was maintained thereafter. ${ }^{31)}$ Radiological comparison using LM scores could not be concluded because of a lack of studies measuring them.

Based on these findings, dupilumab is superior to other biologics in terms of improvements in nasal polyp scores and sense of smell. All biologics, including dupilumab, omalizum$\mathrm{ab}$, and mepolizumab, effectively improved the quality of life.

\section{The present and future of biologics in CRSwNP}

The choice of biologics for CRSwNP often depends on whether a biologic has been approved for use in a country. There is a lack of information on detailed indications of biologics, optimal choices of biologics based on clinical situations, or whether one biologic is more effective than the others. More followup studies are expected to be published in the near future. Furthermore, it may be difficult to use biologics on a wide scale in Korea because the Korean insurance system does not cover them, resulting in financial burden on patients. Additionally, the long-term efficacy of biologics has not been thoroughly examined. Future studies are needed, which elucidate the use of biologics in clinical treatments based on basic immunological research.

As mentioned by the EPOS 2020, the indications for and efficacy of biologics are clinically determined. However, since the prevalence of type 2 inflammation and the histopathological and immunological characteristics of CRSwNP differ between Korea and the Western countries, ${ }^{49)}$ the indications and evaluation criteria proposed by the EPOS 2020 may not be directly applicable in Korea. The evaluation criteria that ac- 
count for the characteristics of type 2 inflammation in the Korean population must be developed. The clinical criteria for a treatment response suggested by the EPOS 2020 are also abstract and ambiguous, and there is no international consensus on how to evaluate the treatment response. Other objective criteria based on blood parameters or endoscopic/radiographic results are needed.

In one of the phase 3 trials, biologics were administered at 2- or 4-week intervals for 24 or 52 weeks. However, it is impractical to apply the same administration period and interval to all patients in an actual clinical situation, because factors-such as symptoms, severity, and compliance-must be considered. A detailed protocol must be established to determine whether the efficacy of biologics remains the same regardless of when it is administered (before or after surgery), the appropriate administration timing, and whether alternating or repeated administration of biologics is possible. It is also essential to establish how to adjust the dose and duration of administration during the maintenance period according to the patient's condition. All these challenges with the use of biologics for CRSwNP would become valuable research topics in the future.

Other immunological mechanisms of biologics, aside from the inhibition of type 2 inflammation, such as the inhibition of type 1 inflammation and regulation of epithelial-derived cytokines (e.g., IL-25, IL-33, and TSLP), as well as B cells that have not undergone $\operatorname{IgE}$ isotype switching may be considered. ${ }^{50)}$ While basic research is actively being conducted on these topics, ${ }^{51-54)}$ further clinical research is needed until the biologics using these mechanisms can be practically used. Whether these biologics can inhibit upstream mechanisms and regulate signaling pathways will be elucidated in the future.

\section{CONCLUSION}

Biologics are being considered as novel treatment options for patients with severe uncontrolled type 2 CRSwNP. Owing to their ability to effectively and safely regulate inflammatory cytokines and cells that mediate type 2 inflammation, biologics are important for patients and rhinologists. Despite the risk of CRSwNP recurrence after cessation of the use of biologics, the lack of detailed information about the indications, administration period, and selection methods of biologics, as well as financial burden for patients, biologics may present a new treatment option once further research is conducted and administrative supplements are made.

This paper reviewed the immunological and clinical findings of biologics currently used to treat rhinologic diseases. We hope efficient use of biologics on severe uncontrolled type 2 CRSwNP that does not respond to medical or surgical treat- ments. In addition, further researches are needed to validate the efficacy and safety of biologics among Korean patients.

\section{Conflicts of Interest}

The authors have no potential conflicts of interest to disclose.

\section{Author Contributions}

Conceptualization: Ji-Hun Mo, Seung-Heon Shin. Data curation: Ki-Il Lee, Gwanghui Ryu, Shin Hyuk Yoo. Formal analysis: Ki-Il Lee. Funding acquisition: Yong Min Kim, Ji-Hun Mo, Seung-Heon Shin. Investigation: Ki-Il Lee, Gwanghui Ryu, Shin Hyuk Yoo. Methodology: Ki-Il Lee. Project administration: Ji-Hun Mo, Seung-Heon Shin. Resources: Ji-Hun Mo, Seung-Heon Shin. Software: Ki-Il Lee. Supervision: Yong Min Kim, Ji-Hun Mo, Seung-Heon Shin. Validation: Gwanghui Ryu, Shin Hyuk Yoo, Yong Min Kim, Ji-Hun Mo, Seung-Heon Shin. Visualization: Ki-Il Lee. Writingoriginal draft: Ki-Il Lee. Writing—review \& editing: Ki-Il Lee, Ji-Hun Mo.

\section{ORCID iDs}

Ki-Il Lee https://orcid.org/0000-0002-2069-7912

Gwanghui Ryu https://orcid.org/0000-0002-3251-399X

Shin Hyuk Yoo https://orcid.org/0000-0002-5083-8720

Yong Min Kim https://orcid.org/0000-0001-5414-8332

Ji-Hun Mo https://orcid.org/0000-0003-1331-364X

Seung-Heon Shin https://orcid.org/0000-0002-9118-0590

\section{Funding Statement}

This research was supported by Basic Science Research Program through the National Research Foundation of Korea (NRF-2020R1A2C1012105 and NRF-2020R1A6A1A03043283). And, this work was supported by Konyang University Myunggok Research Fund of 2019.

\section{REFERENCES}

1) Wang $X$, Cutting GR. Chronic rhinosinusitis. Adv Otorhinolaryngol 2011;70:114-21.

2) Chen $Y$, Dales R, Lin M. The epidemiology of chronic rhinosinusitis in Canadians. Laryngoscope 2003;113(7):1199-205.

3) Hastan D, Fokkens WJ, Bachert C, Newson RB, Bislimovska J, Bockelbrink A, et al. Chronic rhinosinusitis in Europe--an underestimated disease. A GA²LEN study. Allergy 2011;66(9):1216-23.

4) Blackwell DL, Lucas JW, Clarke TC. Summary health statistics for U.S. adults: national health interview survey, 2012. Vital Health Stat 10 2014(260):1-161.

5) Kim YS, Kim NH, Seong SY, Kim KR, Lee GB, Kim KS. Prevalence and risk factors of chronic rhinosinusitis in Korea. Am J Rhinol Allergy 2011;25(3):117-21.

6) Chung JH, Lee YJ, Kang TW, Kim KR, Jang DP, Kim IY, et al. Altered quality of life and psychological health (SCL-90-R) in patients with chronic rhinosinusitis with nasal polyps. Ann Otol Rhinol Laryngol 2015;124(8):663-70.

7) Campbell AP, Phillips KM, Hoehle LP, Feng AL, Bergmark RW, Caradonna DS, et al. Depression symptoms and lost productivity in chronic rhinosinusitis. Ann Allergy Asthma Immunol 2017;118(3):286-9.

8) Kim DW, Cho SH. Emerging endotypes of chronic rhinosinusitis and its application to precision medicine. Allergy Asthma Immunol Res 2017;9(4):299-306.

9) Khan A, Vandeplas G, Huynh TMT, Joish VN, Mannent L, Tomassen P, et al. The Global Allergy and Asthma European Network (GALEN rhinosinusitis cohort: a large European cross-sectional study of chronic rhinosinusitis patients with and without nasal polyps. Rhinology 2019;57(1):32-42.

10) Tomassen P, Vandeplas G, Van Zele T, Cardell LO, Arebro J, Olze H, 
et al. Inflammatory endotypes of chronic rhinosinusitis based on cluster analysis of biomarkers. J Allergy Clin Immunol 2016;137(5): 1449-56.e4.

11) Zhang Y, Gevaert E, Lou H, Wang X, Zhang L, Bachert C, et al. Chronic rhinosinusitis in Asia. J Allergy Clin Immunol 2017;140(5):1230-9.

12) DeConde AS, Mace JC, Levy JM, Rudmik L, Alt JA, Smith TL. Prevalence of polyp recurrence after endoscopic sinus surgery for chronic rhinosinusitis with nasal polyposis. Laryngoscope 2017;127(3):550-5.

13) Alobid I, Antón E, Armengot M, Chao J, Colás C, del Cuvillo A, et al. SEAIC-SEORL. Consensus document on nasal polyposis. POLINA project. J Investig Allergol Clin Immunol 2011;21 Suppl 1:1-58.

14) Rudmik L, Soler ZM. Medical therapies for adult chronic sinusitis: a systematic review. JAMA 2015;314(9):926-39.

15) Gandhi NA, Pirozzi G, Graham NMH. Commonality of the IL-4/IL13 pathway in atopic diseases. Expert Rev Clin Immunol 2017;13(5): 425-37.

16) Ahern S, Cervin A. Inflammation and endotyping in chronic rhinosinusitis-a paradigm shift. Medicina (Kaunas) 2019;55(4):95.

17) Bachert C, Han JK, Wagenmann M, Hosemann W, Lee SE, Backer V, et al. EUFOREA expert board meeting on uncontrolled severe chronic rhinosinusitis with nasal polyps (CRSwNP) and biologics: definitions and management. J Allergy Clin Immunol 2021;147(1):29-36.

18) Wang $X$, Zhang N, Bo M, Holtappels G, Zheng M, Lou H, et al. Diversity of TH cytokine profiles in patients with chronic rhinosinusitis: a multicenter study in Europe, Asia, and Oceania. J Allergy Clin Immunol 2016;138(5):1344-53.

19) Kim SJ, Lee KH, Kim SW, Cho JS, Park YK, Shin SY. Changes in histological features of nasal polyps in a Korean population over a 17year period. Otolaryngol Head Neck Surg 2013;149(3):431-7.

20) Karin J, Tim D, Gabriele H, Cardell LO, Marit W, Claus B. Type 2 inflammatory shift in chronic rhinosinusitis during 2007-2018 in Belgium. Laryngoscope 2021;131(5):E1408-14.

21) Orlandi RR, Kingdom TT, Smith TL, Bleier B, DeConde A, Luong $\mathrm{AU}$, et al. International consensus statement on allergy and rhinology: rhinosinusitis 2021. Int Forum Allergy Rhinol 2021;11(3):213-739.

22) Van Zele T, Gevaert P, Holtappels G, Beule A, Wormald PJ, Mayr S, et al. Oral steroids and doxycycline: two different approaches to treat nasal polyps. J Allergy Clin Immunol 2010;125(5):1069-76.e4.

23) Fokkens WJ, Lund VJ, Hopkins C, Hellings PW, Kern R, Reitsma S, et al. European position paper on rhinosinusitis and nasal polyps 2020 . Rhinology 2020;58(Suppl S29):1-464.

24) Mauer Y, Taliercio RM. Managing adult asthma: the 2019 GINA guidelines. Cleve Clin J Med 2020;87(9):569-75.

25) Gan EC, Habib AR, Hathorn I, Javer AR. The efficacy and safety of an office-based polypectomy with a vacuum-powered microdebrider. Int Forum Allergy Rhinol 2013;3(11):890-5.

26) Bachert C, Zhang L, Gevaert P. Current and future treatment options for adult chronic rhinosinusitis: focus on nasal polyposis. J Allergy Clin Immunol 2015;136(6):1431-40.

27) Bachert C, Zhang N, Cavaliere C, Weiping W, Gevaert E, Krysko O. Biologics for chronic rhinosinusitis with nasal polyps. J Allergy Clin Immunol 2020;145(3):725-39.

28) Kaur D, Hollins F, Woodman L, Yang W, Monk P, May R, et al. Mast cells express IL-13R alpha 1: IL-13 promotes human lung mast cell proliferation and Fc epsilon RI expression. Allergy 2006;61(9):104753.

29) Fujieda S, Matsune S, Takeno S, Ohta N, Asako M, Bachert C, et al. Dupilumab efficacy in chronic rhinosinusitis with nasal polyps from SINUS-52 is unaffected by eosinophilic status. Allergy 2021 May 16 [Epub]. Available from: https://doi.org/10.1111/all.14906.

30) Bachert $C$, Mannent L, Naclerio RM, Mullol J, Ferguson BJ, Gevaert $P$, et al. Effect of subcutaneous dupilumab on nasal polyp burden in patients with chronic sinusitis and nasal polyposis: a randomized clini- cal trial. JAMA 2016;315(5):469-79.

31) Bachert C, Han JK, Desrosiers M, Hellings PW, Amin N, Lee SE, et al. Efficacy and safety of dupilumab in patients with severe chronic rhinosinusitis with nasal polyps (LIBERTY NP SINUS-24 and LIBERTY NP SINUS-52): results from two multicentre, randomised, double-blind, placebo-controlled, parallel-group phase 3 trials. Lancet 2019;394(10209):1638-50.

32) Bachert C, Hellings PW, Mullol J, Hamilos DL, Gevaert P, Naclerio RM, et al. Dupilumab improves health-related quality of life in patients with chronic rhinosinusitis with nasal polyposis. Allergy 2020; 75(1):148-57.

33) Bachert C, Hellings PW, Mullol J, Naclerio RM, Chao J, Amin N, et al. Dupilumab improves patient-reported outcomes in patients with chronic rhinosinusitis with nasal polyps and comorbid asthma. J Allergy Clin Immunol Pract 2019;7(7):2447-9.e2.

34) Jonstam K, Swanson BN, Mannent LP, Cardell LO, Tian N, Wang Y, et al. Dupilumab reduces local type 2 pro-inflammatory biomarkers in chronic rhinosinusitis with nasal polyposis. Allergy 2019;74(4):74352.

35) Takabayashi T, Shigeharu F, Bachert C, Cho SH, Swanson BN, Harel $S$, et al. Dupilumab reduces blood, urine, and nasal biomarkers of type 2 inflammation in patients with chronic rhinosinusitis with nasal polyps in the phase 3 SINUS-52 trial [Abstract]. Proceedings of the 59th Annual Meeting of the Japanese Rhinologic Society; 2020 Oct 10-11; Tokyo, Japan: Japanese Rhinologic Society;2020.

36) Gevaert P, Omachi TA, Corren J, Mullol J, Han J, Lee SE, et al. Efficacy and safety of omalizumab in nasal polyposis: 2 randomized phase 3 trials. J Allergy Clin Immunol 2020;146(3):595-605.

37) Pinto JM, Mehta N, DiTineo M, Wang J, Baroody FM, Naclerio RM. A randomized, double-blind, placebo-controlled trial of anti-IgE for chronic rhinosinusitis. Rhinology 2010;48(3):318-24.

38) Gevaert P, Calus L, Van Zele T, Blomme K, De Ruyck N, Bauters W, et al. Omalizumab is effective in allergic and nonallergic patients with nasal polyps and asthma. J Allergy Clin Immunol 2013;131(1):110-6. el.

39) Bidder T, Sahota J, Rennie C, Lund VJ, Robinson DS, Kariyawasam HH. Omalizumab treats chronic rhinosinusitis with nasal polyps and asthma together-a real life study. Rhinology 2018;56(1):42-5.

40) Mostafa BE, Fadel M, Mohammed MA, Hamdi TAH, Askoura AM. Omalizumab versus intranasal steroids in the post-operative management of patients with allergic fungal rhinosinusitis. Eur Arch Otorhinolaryngol 2020;277(1):121-8.

41) Hayashi H, Mitsui C, Nakatani E, Fukutomi Y, Kajiwara K, Watai K, et al. Omalizumab reduces cysteinyl leukotriene and $9 a, 11 \beta$-prostaglandin F2 overproduction in aspirin-exacerbated respiratory disease. J Allergy Clin Immunol 2016;137(5):1585-7.e4.

42) Han JK, Bachert C, Fokkens W, Desrosiers M, Wagenmann M, Lee SE, et al. Mepolizumab for chronic rhinosinusitis with nasal polyps (SYNAPSE): a randomised, double-blind, placebo-controlled, phase 3 trial. Lancet Respir Med 2021;9:1141-53.

43) Gevaert P, Van Bruaene N, Cattaert T, Van Steen K, Van Zele T, Acke $\mathrm{F}$, et al. Mepolizumab, a humanized anti-IL-5 mAb, as a treatment option for severe nasal polyposis. J Allergy Clin Immunol 2011;128(5): 989-95.el-8.

44) Bachert C, Sousa AR, Lund VJ, Scadding GK, Gevaert P, Nasser S, et al. Reduced need for surgery in severe nasal polyposis with mepolizumab: randomized trial. J Allergy Clin Immunol 2017;140(4):102431.e14.

45) Gevaert P, Lang-Loidolt D, Lackner A, Stammberger H, Staudinger H, Van Zele T, et al. Nasal IL-5 levels determine the response to antiIL-5 treatment in patients with nasal polyps. J Allergy Clin Immunol 2006;118(5):1133-41.

46) Bachert C, Desrosiers M, Gevaert P, Heffler E, Hopkins C, Tversky 
JR, et al. Efficacy and safety of benralizumab for the treatment of chronic rhinosinusitis with nasal polyps: results from the phase III OSTRO trial. Proceedings of the EAACI Hybrid Congress 2021; 2021 Jul 10-12; Krakow, Republic of Poland: EAACI;2021.

47) Hellings PW, Verhoeven E, Fokkens WJ. State-of-the-art overview on biological treatment for CRSwNP. Rhinology 2021;59(2):151-63.

48) Peters AT, Han JK, Hellings P, Heffler E, Gevaert P, Bachert C, et al. Indirect treatment comparison of biologics in chronic rhinosinusitis with nasal polyps. J Allergy Clin Immunol Pract 2021;9(6):2461-71.e5.

49) Kim DW. Can neutrophils be a cellular biomarker in Asian chronic rhinosinusitis? Clin Exp Otorhinolaryngol 2019;12(4):325-6.

50) Wang H, Pan L, Liu Z. Neutrophils as a protagonist and target in chronic rhinosinusitis. Clin Exp Otorhinolaryngol 2019;12(4):337-47.

51) Vannella KM, Ramalingam TR, Borthwick LA, Barron L, Hart KM, Thompson RW, et al. Combinatorial targeting of TSLP, IL-25, and IL-
33 in type 2 cytokine-driven inflammation and fibrosis. Sci Transl Med 2016;8(337):337ra65.

52) Shin HW, Kim DK, Park MH, Eun KM, Lee M, So D, et al. IL-25 as a novel therapeutic target in nasal polyps of patients with chronic rhinosinusitis. J Allergy Clin Immunol 2015;135(6):1476-85.e7.

53) Nagarkar DR, Poposki JA, Tan BK, Comeau MR, Peters AT, Hulse KE, et al. Thymic stromal lymphopoietin activity is increased in nasal polyps of patients with chronic rhinosinusitis. J Allergy Clin Immunol 2013;132(3):593-600.e12.

54) Hernandez ML, Mills K, Almond M, Todoric K, Aleman MM, Zhang $\mathrm{H}$, et al. IL-1 receptor antagonist reduces endotoxin-induced airway inflammation in healthy volunteers. J Allergy Clin Immunol 2015; 135(2):379-85.

55) Laidlaw TM, Buchheit KM. Biologics in chronic rhinosinusitis with nasal polyposis. Ann Allergy Asthma Immunol 2020;124(4):326-32. 